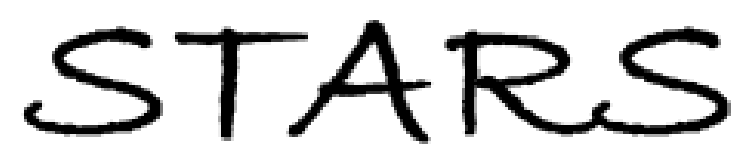

University of Central Florida

STARS

$1-1-2007$

\title{
Anisotropic optical phonon scattering of holes in cubic semiconductors
}

\author{
M. V. Dolguikh \\ University of Central Florida \\ R. E. Peale \\ University of Central Florida
}

Find similar works at: https://stars.library.ucf.edu/facultybib2000 University of Central Florida Libraries http://library.ucf.edu

This Article is brought to you for free and open access by the Faculty Bibliography at STARS. It has been accepted for inclusion in Faculty Bibliography 2000 s by an authorized administrator of STARS. For more information, please contactSTARS@ucf.edu.

\section{Recommended Citation}

Dolguikh, M. V. and Peale, R. E., "Anisotropic optical phonon scattering of holes in cubic semiconductors" (2007). Faculty Bibliography 2000s. 7062.

https://stars.library.ucf.edu/facultybib2000/7062

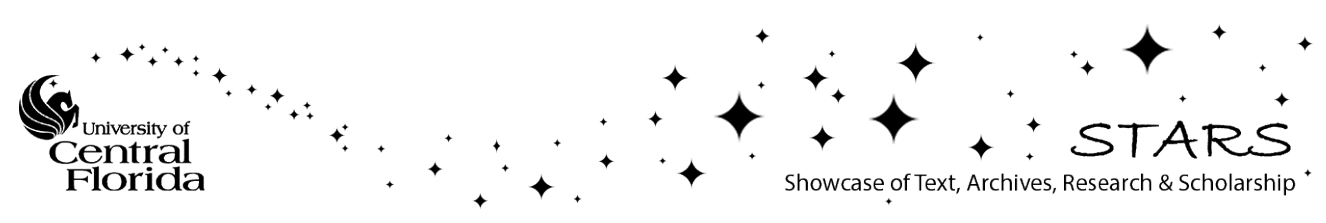




\section{Anisotropic optical phonon scattering of holes in cubic semiconductors}

Cite as: J. Appl. Phys. 101, 113716 (2007); https://doi.org/10.1063/1.2745222

Submitted: 05 March 2007. Accepted: 18 April 2007. Published Online: 15 June 2007

M. V. Dolguikh, and R. E. Peale

\section{ARTICLES YOU MAY BE INTERESTED IN}

Electrical transport in $\mathrm{n}$-type $4 \mathrm{H}$ silicon carbide

Journal of Applied Physics 90, 1869 (2001); https://doi.org/10.1063/1.1382849

Nitrogen donors in $4 \mathrm{H}$-silicon carbide

Journal of Applied Physics 73, 3332 (1993); https://doi.org/10.1063/1.352983

Monte Carlo study of hole mobility in Al-doped $4 \mathrm{H}-\mathrm{SiC}$

Journal of Applied Physics 91, 1359 (2002); https://doi.org/10.1063/1.1429802

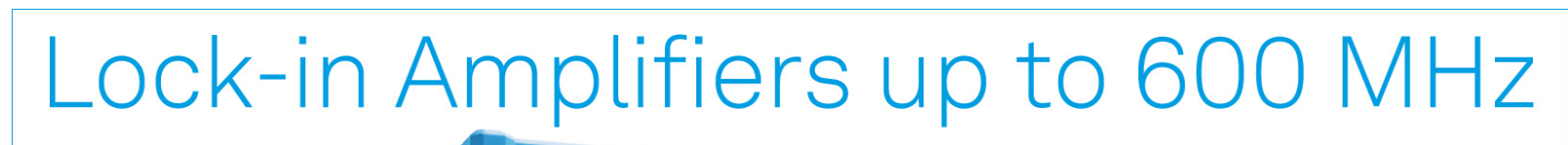

starting at

$\$ 6,210$

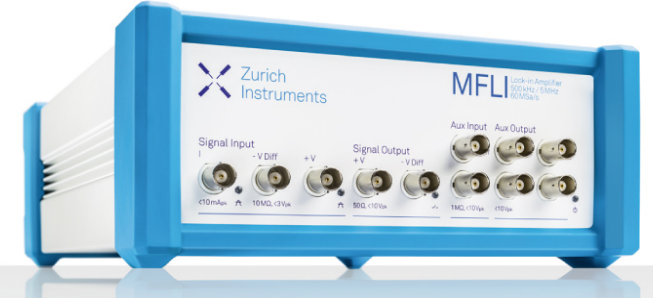

J. Appl. Phys. 101, 113716 (2007); https://doi.org/10.1063/1.2745222

(c) 2007 American Institute of Physics.
101, 113716 


\title{
Anisotropic optical phonon scattering of holes in cubic semiconductors
}

\author{
M. V. Dolguikh and R. E. Peale ${ }^{a)}$ \\ Department of Physics, University of Central Florida, Orlando, Florida 32816
}

(Received 5 March 2007; accepted 18 April 2007; published online 15 June 2007)

\begin{abstract}
The formula for the nonpolar optical phonon scattering rate of holes in cubic semiconductors is obtained in the case of strong valence band anisotropy. The deformation potential approximation is used. A three-band, $6 \times 6, \mathbf{k} \cdot \mathbf{p}$ Luttinger-Kohn representation includes states belonging to the heavy, light, and split-off bands. Mixing with the latter causes strong anisotropy in the transition matrix elements as well as in the density of final states. The derived formula is recommended for silicon, where inter- and intravalence-band scattering rates are much more strongly anisotropic and have significantly different values than those estimated from the usual two-band $4 \times 4$, "warped spheres" approximation that neglects the split-off band. Results for the more isotropic case of germanium are presented for comparison. (C) 2007 American Institute of Physics. [DOI: 10.1063/1.2745222]
\end{abstract}

\section{INTRODUCTION}

Scattering of holes in semiconductors is induced by emission or absorption of phonons (acoustic and optical), scattering on impurities (ionized and neutral), and scattering on mobile carriers. A general discussion of different scattering mechanisms in $p$-type semiconductors is found in Refs. 1-3. Rate formulas for each type of scattering have been derived in separate papers by different authors. ${ }^{4-7}$ Clearly, any accurate description of transport or device performance must consider all mechanisms simultaneously.

The description of each scattering phenomenon remains incomplete, as published rate formulas have limited applicability. Recently, it was found necessary to develop more accurate formulas for hole-hole scattering ${ }^{7}$ to accurately simulate hot-hole dynamics for $p$-Ge intervalence-band laser structures at high hole concentrations. ${ }^{8,9}$ For $p$-Si, a potential hot-hole terahertz gain medium, ${ }^{10-12}$ published rate formulas ${ }^{1,12,13}$ incompletely account for the strong valenceband anisotropy by neglecting the split-off band. Mixing with this band strongly affects hole wave functions, the scattering matrix elements, and the density of states.

In the case of acoustic phonon scattering, the rate increases with temperature. Since acoustic phonons also absorb terahertz radiation, they limit the maximum hot-hole laser operating temperature and duty cycle. Yet anisotropic scattering rate formulas for $p$-Si are lacking in the literature. Fortunately, acoustic phonon scattering can be neglected in initial zeroth-order simulations because it is frozen out at low temperatures.

Ionized impurity and hole-hole scattering are important even at low temperature. These limit the maximum useful concentration of holes, and therefore the maximum achievable hot-hole laser gain. The recently published ${ }^{7}$ hole-hole scattering rate formulas are accurate for $p$-Si. Impurity scattering is similar to hole-hole scattering, where one of the particles is fixed.

Optical phonon scattering involves large energy ex-

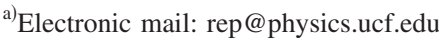

change, dominates hot-carrier transport, and is responsible for generating population inversion in hot-hole semiconductor lasers. ${ }^{14-16}$ Accurate optical phonon scattering rates are of primary importance for numerical simulation and optimization of intervalence-band hot-hole lasers based on $p$-type $\mathrm{Ge},{ }^{14-16} \mathrm{Si}^{12}{ }^{12} \mathrm{GaAs},{ }^{17}$ the negative mass cyclotron-resonance maser, ${ }^{18,19}$ and other devices. The slow holes produced after optical phonon emission are especially vulnerable to holehole and ionized impurity scattering, ${ }^{7}$ simulation of which demands accurate directional distributions. Traditional formulas for optical phonon scattering ${ }^{1,12}$ fail in providing such distributions for $p$-Si by neglecting the split-off band and the resulting anisotropy. In this paper, the optical phonon scattering rate formula in the case of strong valence band anisotropy is obtained and discussed.

When the optical phonon energy is much smaller than spin-orbit splitting (e.g., for Ge and GaAs), mixing of lightand heavy-hole states with split-off band states is safely neglected. Then, optical phonon scattering of holes is treated in the "warped spheres" approximation $(4 \times 4 \mathbf{k} \cdot \mathbf{p}$ model $)$ with parabolic hole dispersion law. ${ }^{20}$ This simplification is suitable for Ge and GaAs, but is inapplicable to silicon, whose optical phonon scattering threshold $\hbar \omega_{\text {op }}=61 \mathrm{meV}$ exceeds the spin-orbit splitting $\Delta=44 \mathrm{meV}$. For silicon, mixing with the split-off band states significantly alters the scattering rate and directional dependence. Due to the complexity of this problem, past authoritative treatments have elected to consider optical phonon scattering in all cubic semiconductors, including silicon, in a basis that excludes the split-off band. ${ }^{1,12}$ We demonstrate here the poorness of this approximation for $p$-Si.

Drift velocities and hole current in crossed electric and magnetic fields at low temperatures in $p$-Si have been calculated previously, ${ }^{21}$ where authors claimed to incorporate anisotropy of optical phonon scattering in a Monte Carlo simulation, but presentation or citation of a rate formula was lacking, so that it was unclear whether anisotropy in both transition matrix element and density of final states were accounted for. The transition matrix elements for some selected high-symmetry directions in germanium have been 
previously calculated with inclusion of split-off band states, ${ }^{22}$ but results for arbitrary directions were absent. Thus, a presentation of an explicit rate formula for the general case of optical phonon scattering in the three-band, $6 \times 6$ basis, which includes admixture of the split-off band states into light- and heavy-hole states, appears to be lacking. This paper presents explicit matrix elements and total scattering rates for arbitrary directions in cubic semiconductors in the three-band model, together with a comparative discussion for $p$-Si and the more isotropic $p$-Ge.

An immediate application is accurate simulation of hothole dynamics in silicon in order to calculate and optimize gain in a $p$-Si terahertz laser, which has potential advantages over the two established semiconductor terahertz lasers, namely, the $p$-Ge laser ${ }^{14}$ and AlGaAs-based quantum cascade lasers. $^{23}$ Those lasers operate only up to frequencies of about $4.5 \mathrm{THz}$ due to the onset of strong terahertz absorption by phonons, which increases rapidly with frequency and temperature. In contrast, $\mathrm{Si}$ has high transparency up to $10 \mathrm{THz}$, and the temperature dependence of the lattice absorption is comparatively weak. ${ }^{24}$ In addition to the better material properties for high terahertz and high temperature operation, a $\mathrm{Si}$ terahertz laser might be integrated with $\mathrm{Si}$ optoelectronics.

An early Monte Carlo investigation of hot-hole lasing in $p$-Si reported results for orientations $(\mathbf{B}, \mathbf{E})=(111,11-2)$ and (001, 100) only. ${ }^{25}$ A classical calculation of the volume of light-hole accumulation in momentum space has suggested maximal spontaneous emission for low temperature $p$-Si in crossed electric and magnetic fields with orientations (001, 1-10) and $(11-2,111){ }^{26}$ A second Monte Carlo study ${ }^{12}$ found terahertz amplification for $(001,100)$ and $(001,110)$, but not for $(110,1-10)$. The first (and never repeated) experimental observation $^{27}$ of stimulated emission in $p$-Si was limited to the orientation $(11-2,1-10)$ and found weak signal in the range of $28-40 \mathrm{~cm}^{-1}$. Quantum mechanical calculations of light-hole Landau level lifetimes have been performed only for the orientations (110,1-10) and (001,1-10), for which the Hamiltonian simplifies. ${ }^{28}$ A subsequent experiment, ${ }^{29}$ which was limited to applied field orientations $(001,100)$, reported terahertz emission above $50 \mathrm{~cm}^{-1}$. Clearly, neither theoretical nor experimental possibilities have been thoroughly explored, and in some cases the theoretical predictions are contradictory. Accurate predictions require the formula presented here.

A possible future application of the result presented here is simulation of hot-hole transport in $p$-type diamond. In diamond, the spin-orbit splitting (6 meV) (Ref. 1) is much smaller than in silicon, and the optical phonon scattering threshold (167 meV) (Ref. 1) is much higher. Thus, the effects of valence band anisotropy are expected to be more severe. High-field hole-drift velocity in natural diamond has been measured, but the theory used a two-band Monte Carlo simulation that did not allow interpretation of the observed anisotropy. $^{30}$

\section{SCATTERING RATE FORMULA}

Equation (1) presents the total transition rate with absorption (upper part) or emission (lower part) of an optical phonon. The derivation is presented in the Appendix.

$$
\begin{aligned}
P\left(\mathbf{k}, \nu, \nu^{\prime}\right)= & \frac{1}{8 \pi^{2} \rho \omega_{\mathrm{op}}}\left\|\bar{n}_{\hbar \omega_{\mathrm{op}}}+1\right\| \int \frac{k^{\prime 2}}{\left[\partial E_{\nu^{\prime}}\left(k^{\prime}, \Omega^{\prime}\right) / \partial k^{\prime}\right]_{\Omega^{\prime}}} \\
& \times R_{\mathrm{opt}}\left(\mathbf{k}, \nu ; \nu^{\prime}, \Omega^{\prime}\right) d \Omega^{\prime},
\end{aligned}
$$

where

$$
R_{\mathrm{opt}}\left(\mathbf{k}, \nu ; \nu^{\prime}, \Omega^{\prime}\right)=\frac{1}{2} \sum_{\sigma, \sigma^{\prime}=+,-} \sum_{\mathbf{e}^{s}}\left|\left\langle\mathbf{k}^{\prime}, \nu^{\prime \sigma^{\prime}}\left|H_{\mathrm{opt}}\left(\mathbf{e}^{s}\right)\right| \mathbf{k}, \nu^{\sigma}\right\rangle\right|^{2},
$$

with $\mathbf{k}^{\prime}=\mathbf{k}^{\prime}\left(k^{\prime}, \Omega^{\prime}\right)$, and magnitude $k^{\prime}$ is subject to condition [Eq. (A16)]. In Eq. (1) $\rho$ is the mass density, $\omega_{\text {op }}$ the optical phonon frequency, $\bar{n}_{\hbar \omega_{\mathrm{op}}}$ the Bose-Einstein phonon distribution, $E_{\nu^{\prime}}$ is the energy of the final-state band $\nu^{\prime}$, and we integrate over the direction of the final-state hole wave vector $\mathbf{k}^{\prime}$. In Eq. (2) $H_{\text {opt }}$ is the deformation potential operator, we sum over the three orthogonal phonon polarization vectors $\mathbf{e}^{s}{ }^{13}$ sum over final effective spin projections $\sigma^{\prime}$, and average over initial effective spin projections $\sigma$. Equation (2) depends on both initial and final directions of the hole. When this anisotropic squared matrix element is multiplied by the anisotropic density of the final states, as in the integrand of Eq. (1), the anisotropy of the total scattering rate is determined. The integrand of Eq. (1) defines the differential scattering rate of the hole with wave vector $\mathbf{k}$ from band $\nu$ to band $\nu^{\prime}$ into the direction $\Omega^{\prime}$. Using well-known hole wave functions [Eqs. (A8) and (A9)], all possible intra- and intervalence-band transition rates between light and heavy bands with absorption or emission of an optical phonon can be found for an arbitrary initial hole state in the anisotropic and nonparabolic three-band model.

Optical phonon scattering in cubic semiconductors ${ }^{1,12,13}$ usually is considered in the warped spheres, $4 \times 4$, two-band approximation. See Eqs. (A10)-(A12) for energy spectrum and wave functions. This achieves great analytic simplification. We repeat the simplified scattering rate formula here for completeness. Neglecting the split-off band, Eq. (2) becomes $^{20}$

$$
R_{\mathrm{opt}}^{\mathrm{warp}}\left(\mathbf{k}, \nu ; \nu^{\prime}, \Omega^{\prime}\right)=d_{\mathrm{opt}}^{2} \Psi_{\mathbf{k} \mathbf{k}^{\prime}}^{\nu \nu^{\prime}}+\sum_{\mathbf{e}^{s}} \delta E_{\nu}\left(\mathbf{k}, \mathbf{e}^{s}\right) \delta E_{\nu^{\prime}}\left(\mathbf{k}^{\prime}, \mathbf{e}^{s}\right),
$$

where 


$$
\Psi_{\mathbf{k} \mathbf{k}^{\prime}}^{\nu v^{\prime}}=\frac{1}{2}\left\{1 \mp \frac{D^{2}\left(\mathbf{k} \cdot \mathbf{k}^{\prime}\right)-B^{2} k^{2} k^{\prime 2}+\left(3 B^{2}-D^{2}\right) \sum_{i} k_{i}^{2} k_{i}^{\prime 2}}{2 \sqrt{\left[B^{2} k^{\prime 4}+C^{2}\left(k_{x}^{\prime 2} k_{y}^{\prime 2}+k_{x}^{\prime 2} k_{z}^{\prime 2}+k_{y}^{\prime 2} k_{z}^{\prime 2}\right)\right]\left[B^{2} k^{4}+C^{2}\left(k_{x}^{2} k_{y}^{2}+k_{x}^{2} k_{z}^{2}+k_{y}^{2} k_{z}^{2}\right)\right]}}\right\},
$$

and

$$
\delta E_{H, L}\left(\mathbf{k}, \mathbf{e}^{s}\right)= \pm \frac{D d_{\mathrm{opt}}\left(k_{x} k_{y} e_{z}^{s}+k_{y} k_{z} e_{x}^{s}+k_{x} k_{z} e_{y}^{s}\right)}{\sqrt{B^{2} k^{4}+C^{2}\left(k_{x}^{2} k_{y}^{2}+k_{x}^{2} k_{z}^{2}+k_{y}^{2} k_{z}^{2}\right)}}
$$

The upper (lower) sign in Eq. (4) holds for intra(inter-)valence-band transitions. The upper (lower) sign in (5) holds for the heavy-(light)-hole band. Using parabolic dispersion [Eq. (A10)], integral (1) simplifies to

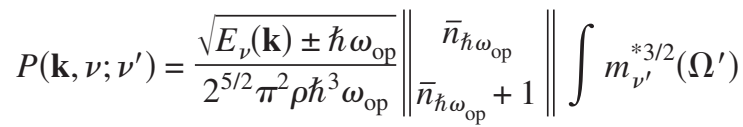

$$
\begin{aligned}
& \times R_{\mathrm{opt}}^{\mathrm{warp}}\left(\mathbf{k}, \nu ; \nu^{\prime}, \Omega^{\prime}\right) d \Omega^{\prime},
\end{aligned}
$$

where $m_{\nu}^{*}(\Omega)=\hbar^{2} k^{2} / 2 E_{\nu}(k, \Omega)$ is the effective mass of the hole within the $\nu$ th band in the $\Omega$ direction. The effective mass is independent of the magnitude of $\mathbf{k}$ in this approximation.

\section{DISCUSSION}

Squared matrix elements Eqs. (2) and (3), and scattering rates Eqs. (1) and (6), were evaluated numerically using values for the parameters $\left\{L, M, N, \Delta, \hbar \omega_{\mathrm{op}}\right\}$ of $\{-30.4,-5.7$, $-33.9,290,37\}$ for germanium and $\{-6.5,-3.6,-8.7,44,61\}$ for silicon (see Appendix). ${ }^{31}$ The units for valence band parameters $L, M$, and $N$ are $\hbar^{2} / 2 m_{e}$. The spin-orbit splitting $\Delta$ and optical phonon energy $\hbar \omega_{\mathrm{op}}$ are in meV.

To demonstrate the effect of including the split-off band on the transition matrix elements, squared matrix element Eq. (2) for the case of emission of an optical phonon in $\mathrm{Si}$ is plotted in Figs. 1 and 2 for different initial hole energies and all possible intra- and intervalence-band transitions as a function of the final direction of the scattered hole. For these examples, the hole is chosen to move in the $\langle 100\rangle$ direction initially, as indicated by the arrows. The prominent lobes in the horizontal plane in the lower right diagram in Fig. 1 are all in the $\langle 110\rangle$ directions, and all other diagrams in Figs. 1 and 2 are similarly oriented. Left (right) columns represent intra-(inter-)valence-band transitions for the hole initially in the heavy (Fig. 1) or light (Fig. 2) band. The top row in each figure presents results calculated from Eq. (3) in the twoband approximation, where the matrix element is independent of the initial hole energy, whose spectrum is parabolic. The three lower rows in each figure are results from Eq. (2) for the full three-band model [see Eqs. (A7)-(A9)]. In this approximation the shape of the surface depends on the initial energy of the hole, so results are plotted for three energy values $1.1 \hbar \omega_{\mathrm{op}}, 1.5 \hbar \omega_{\mathrm{op}}$, and $2.0 \hbar \omega_{\mathrm{op}}$. The matrix element becomes highly anisotropic as energy increases, and in the case of an initially light hole (Fig. 2) it also decreases in magnitude as energy grows (all surfaces are plotted to scale).
We next demonstrate the effect of split-off band inclusion on the direction dependence of the scattering. Figure 3 presents differential intra- and intervalence-band transition rates with emission of an optical phonon, as defined by the integrand of Eq. (1) where the squared matrix element Eq. (2) is multiplied by the density of final states, as a function of final hole direction in germanium and silicon. Initial energies are taken to be $2 \hbar \omega_{\text {op }}(74 \mathrm{meV}$ for $\mathrm{Ge}$ and $122 \mathrm{meV}$ for $\mathrm{Si}$ ),

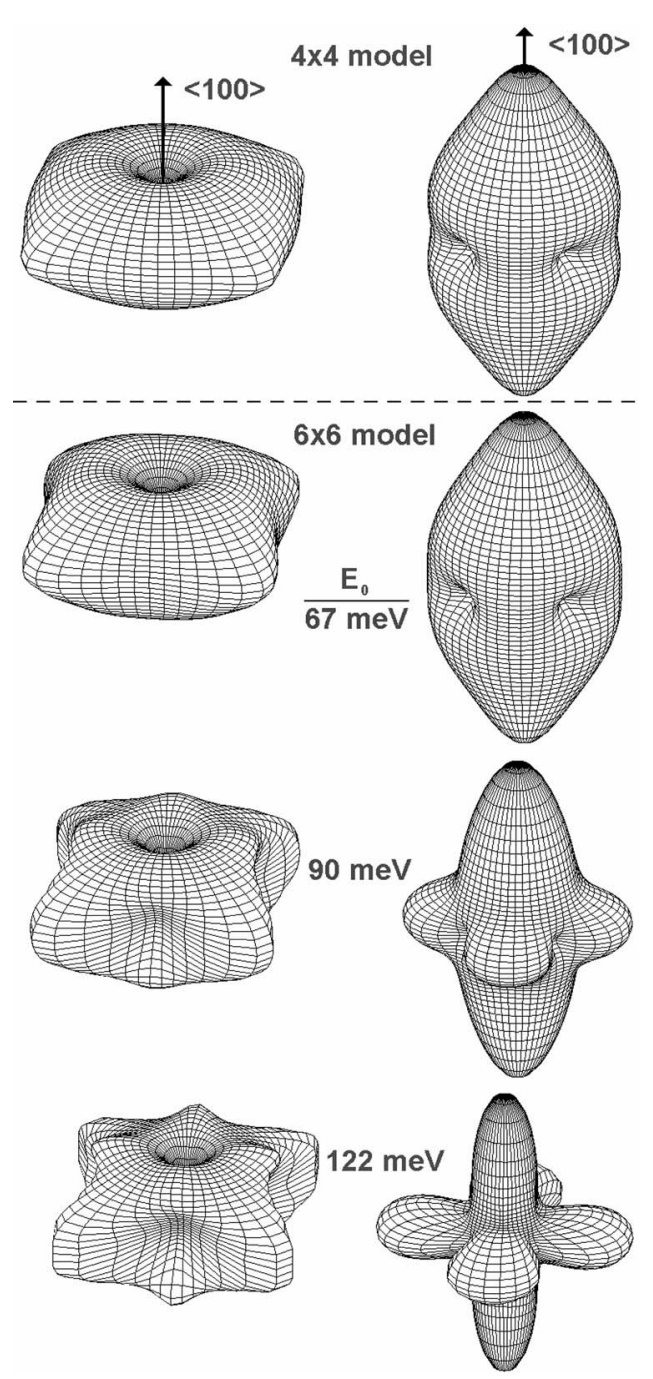

FIG. 1. Comparison of three-band model and two-band approximation matrix elements for optical phonon scattering of heavy holes in silicon. (Top row) The distance of the surface from the origin is proportional to the squared matrix element Eq. (3) for intra- $(H \rightarrow H$, left $)$ and inter- $(H \rightarrow L$, right)valence-band transitions with emission of an optical phonon calculated in the two-band approximation as a function of final hole direction. (Lower three rows) Squared matrix element Eq. (2) $H \rightarrow H$, left) and inter- $(H \rightarrow L$, right)valence-band transitions with emission of an optical phonon calculated in the three-band model as a function of hole energy and final hole direction. The hole is initially moving in the $\langle 100\rangle$ direction as indicated by the arrows. 


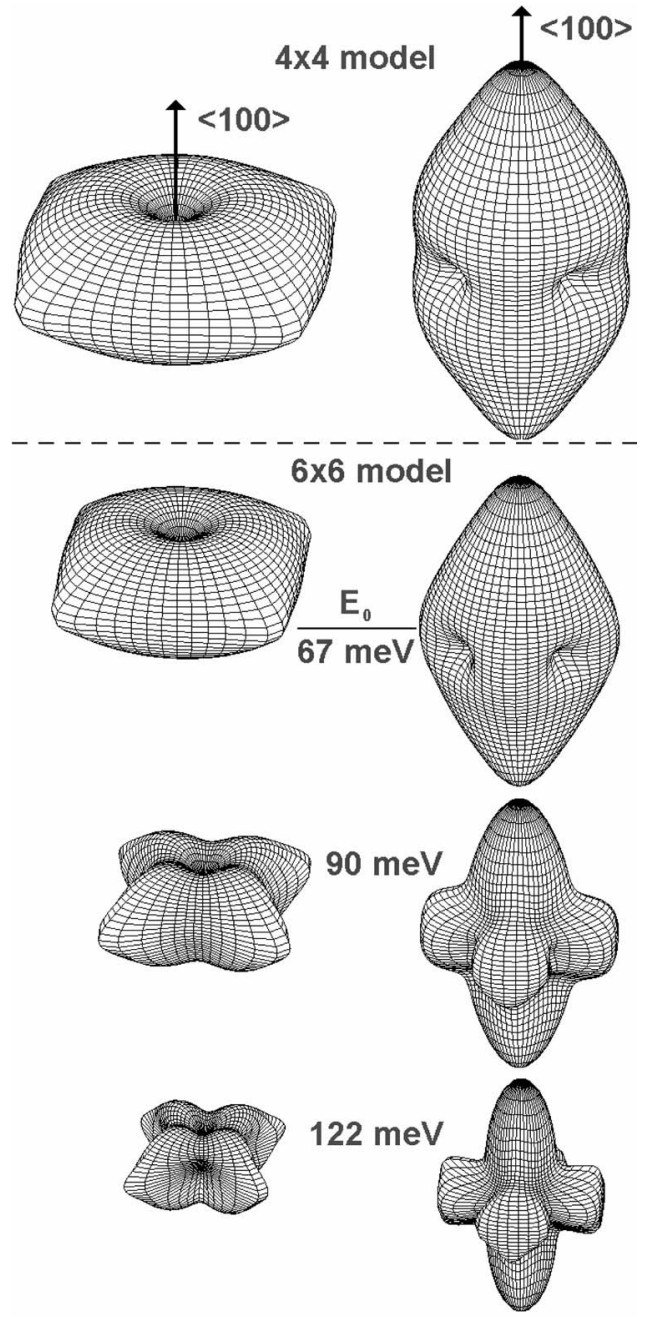

FIG. 2. Comparison of three-band model and two-band approximation matrix elements for scattering of light holes in silicon. The layout is the same as in Fig. 1 except that the left column is for $L \rightarrow L$ and the right column for $L \rightarrow H$ transitions.

and the chosen initial direction of the hole is $\langle 100\rangle$ as indicated by the arrows. The scale of each surface is chosen differently for clarity. The plotted surfaces are directional diagrams for hole scattering with the chosen initial direction and energy. Such results should be used for the statistical selection of the final direction of the scattered hole in numerical simulations. The anisotropy is clearly very much stronger for $\mathrm{Si}$ than for $\mathrm{Ge}$. In contrast, the two-band approximation gives differential rates for $\mathrm{Si}$ and $\mathrm{Ge}$ that have very similar angular dependence, which is very close to the three-band model results for Ge in Fig. 3.

The effect of the initial hole direction on the total scattering rate is discussed next. When integrated over all possible final directions, the scattering rate Eq. (1) is still a strong function of initial direction in the case of silicon in the three-band model. Figures 4 and 5 present scattering rate Eq. (1) plotted for all possible intra- (light-to-light and heavy-toheavy) and inter- (heavy-to-light and light-to-heavy)valenceband transitions for a hole initially moving along the main crystallographic directions $(\langle 100\rangle,\langle 110\rangle$, and $\langle 111\rangle)$ in $\mathrm{Si}$, as a function of initial hole energy. The rates are plotted in the low temperature limit $\left(\bar{n}_{\hbar \omega_{\mathrm{op}}} \ll 1\right)$, so that only scattering with

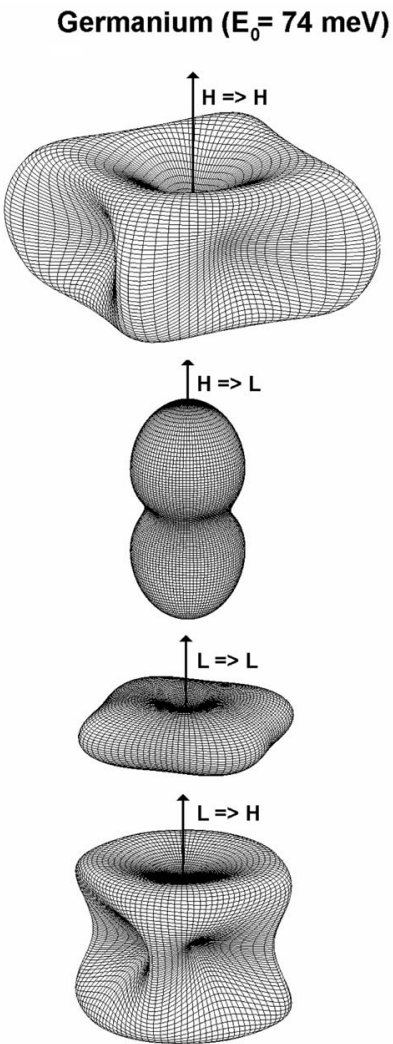

Silicon $\left(E_{0}=122 \mathrm{meV}\right)$


FIG. 3. Comparison of differential optical phonon scattering rates for holes in Ge and Si. Diagrams represent the directional distribution of scattered holes, and the distance from the origin to the surface is proportional to the scattering rate. Arrows indicate the initial $\langle 100\rangle$ hole direction and the viewpoint is the same as in Figs. 1 and 2.

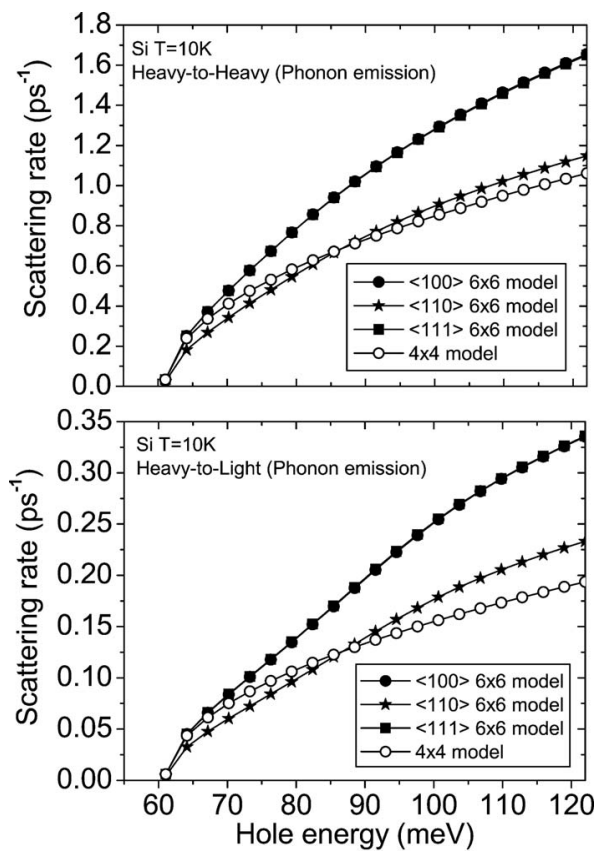

FIG. 4. Heavy-hole scattering rate spectrum. The intra-(top) and intervalence-band (bottom) total transition rates for scattering of heavy holes with emission of an optical phonon in $\mathrm{Si}$ as a function of hole energy. The different initial directions are indicated in the legend. The rate in the twoband approximation, which is independent of intial hole direction, is plotted for comparison 

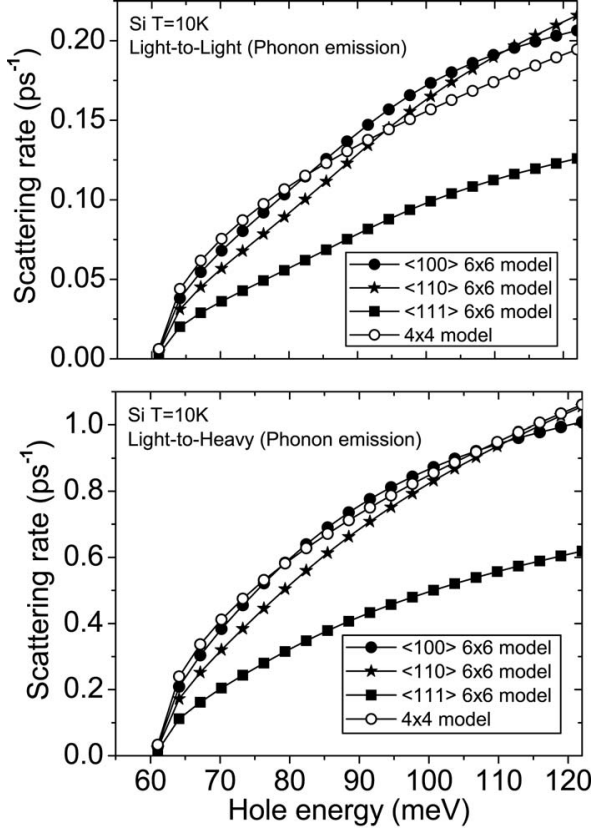

FIG. 5. Light-hole scattering rate spectrum. The organization of the plots is the same as in Fig. 4

emission of an optical phonon occurs. The rates have a threshold at the optical phonon energy $61 \mathrm{meV}$. For heavyto-heavy transitions (Fig. 4), the rates along directions $\langle 100\rangle$ and $\langle 111\rangle$ are indistinguishable on this scale, and the same holds for heavy-to-light transitions. The rate Eq. (6) in the two-band approximation is plotted for comparison in Figs. 4 and 5 , and it is independent of the initial direction of the hole.

The dependence of the scattering rate Eq. (1) on initial direction for silicon is illustrated in Fig. 6 for a fixed initial hole energy of $90 \mathrm{meV}$. The viewpoint and crystallographic directions are the same as in Figs. 1-3. The angular dependences for intra- and intervalence-band transitions with the same initial band are similar (see Figs. 4 and 5), so that one diagram serves for both rates but with different scales. For the $\langle 100\rangle$ initial direction, the scattering rate is about 1.5 times higher than for the $\langle 110\rangle(\langle 111\rangle)$ initial direction for heavy (light) holes. This ratio is increased when the incident hole becomes faster. Anisotropy in the same total scattering rates calculated for germanium is negligible.

\section{CONCLUSIONS}

The rates for inter- and intravalence-band transitions caused by emission or absorption of an optical phonon are characterized by two factors. First is the matrix-element anisotropy. When mixing with the split-off band is included (three-band model), matrix-element anisotropy is very strong for silicon and increases with hole energy. When split-off band states are excluded (two-band approximation), the matrix element lacks strong anisotropy and has no energy dependence. Therefore, the two-band approximation is poor for $\mathrm{Si}$, though it has been widely used. The second factor is the density of states in the final band. The integrated density of states is significantly larger when split-off band states are included. The ratio between these densities grows rapidly

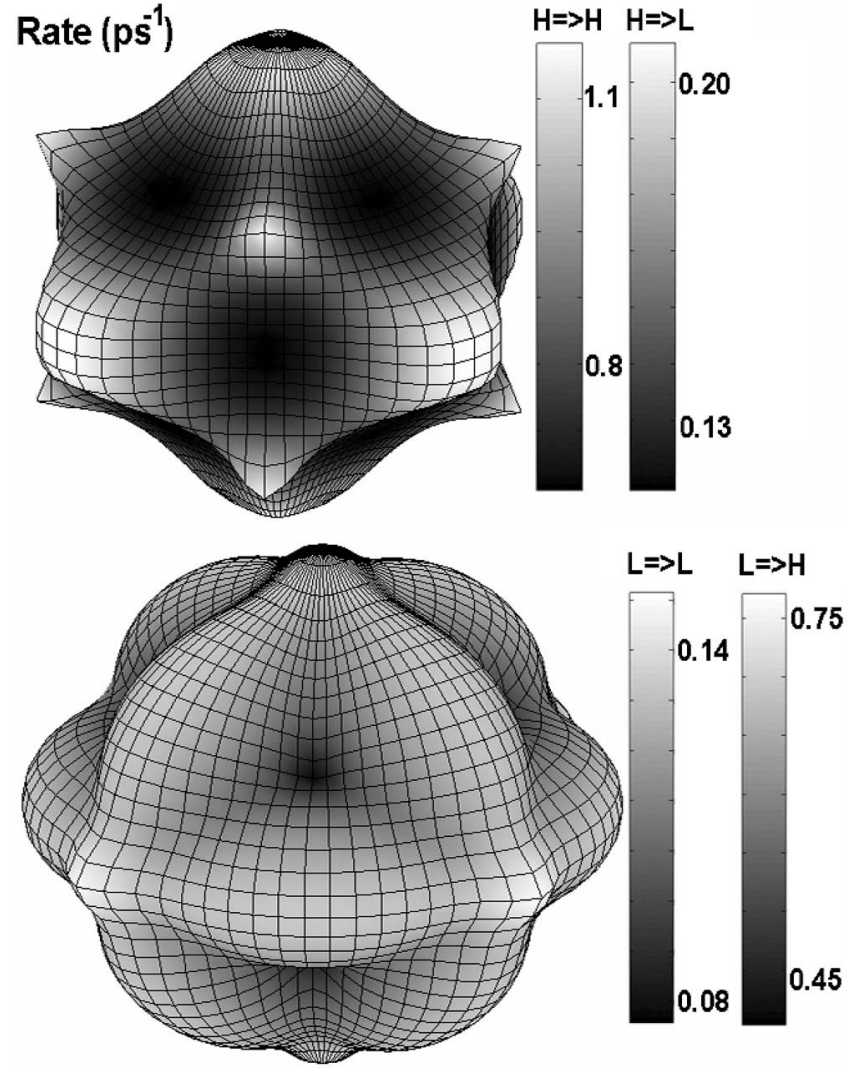

FIG. 6. Total (integrated over final states) optical phonon rate for holes in $\mathrm{Si}$ plotted as a function of initial direction of the hole with energy of $90 \mathrm{meV}$. The distance of the surface from the origin is proportional to the rate, while the gray scale bars indicate actual rate values in units of $\mathrm{ps}^{-1}$. The $\langle 100\rangle$ directions correspond to the large rounded lobes on top and on the lower sides of the upper diagram, while the three dark pits are the $\langle 110\rangle$ directions in this diagram. The same point of view applies to the lower diagram, where the central dark pit is a $\langle 111\rangle$ direction.

with energy. ${ }^{10}$ For some special initial hole directions, this increase is compensated by a decrease in the matrix element, and the total rate in the three-band model approaches that found in the two-band approximation. Use of the two-band approximation matrix element together with the fully anisotropic density of the final states, as was done in Ref. 12, overestimates the total scattering rate in $\mathrm{Si}$ by a factor of up to $\sim 2(\sim 3)$ for heavy (light) holes.

For accurate calculation of optical phonon scattering in silicon, split-off band states must be included when determining both density of states and transition matrix element. The usual two-band approximation ${ }^{1,13}$ is unsuitable for semiconductors with small spin-orbit splitting because it fails to describe accurately the anisotropy of the integrated scattering rate, revealed for silicon in Fig. 6. However, exclusion of split-off band states is justified for semiconductors with large spin-orbit splitting, such as Ge or GaAs.

Anisotropy of the integrated optical phonon scattering rate for $\mathrm{Si}$ is important in choosing the best light- and heavyhole streaming directions for intervalence-band $p$-Si lasers. ${ }^{10,11}$ For this type of device, optical phonon scattering is the dominant source of inversion-building heavy-to-light transitions. Because of the strong anisotropy of light- and heavy-hole streaming in crossed fields and hole-hole scattering anisotropy, ${ }^{7}$ correct calculation of the directional distri- 
bution of scattered holes is very important for correct estimation of hole lifetimes. The preferable direction of the heavy-hole streaming (in terms of highest light-hole generation rate) would be $\langle 100\rangle$ or $\langle 111\rangle$ (see Figs. 4-6). At the same time it is favorable for the light-hole population to have the fastest part of the trajectory in the $\langle 111\rangle$ direction, since then the light-to-heavy hole transitions by optical phonon emission are minimized. Because of the complexity of the problem, Monte Carlo simulation using three-band model scattering rates can help to optimize the laser performance. As was shown above, simulation with simplified approximation can give wrong predictions.

\section{ACKNOWLEDGMENTS}

The authors thank Dr. Andrey Muravjov for useful discussions. This work was supported in part by Grant No. FA871805C0078 from AFRL/SNHC at Hanscom AFB and by an ASEE/AFOSR summer faculty fellowship to one of the authors (R.E.P.) in 2005.

\section{APPENDIX}

Spin-orbit coupling splits the sixfold degenerate valence band at hole wave vector $\mathbf{k}=0$ for semiconductors with diamond or zinc-blende structure into a fourfold degenerate band (light and heavy bands) and a twofold degenerate band (split-off band), which is shifted toward higher hole energy. If both spin-orbit splitting at $\mathbf{k}=0$ and hole kinetic energy are small compared to the fundamental band gap, the wave functions of light- and heavy-hole states, to lowest order in $k$, can be written as ${ }^{20}$

$$
\psi^{\nu}(\mathbf{k}, \mathbf{r})=\exp (i \mathbf{k} \cdot \mathbf{r}) \sum_{J=3 / 2,1 / 2} \sum_{m_{J}=-J}^{J} c_{J, m_{J}}^{\nu}(\mathbf{k}) \psi_{m_{J}}^{J}(\mathbf{r})
$$

Here, $\nu$ is the band index (heavy, light, or split-off). The $\psi_{m}^{J}$ are basis functions in the Luttinger-Kohn representation ${ }^{20,32}$ given by

$$
\begin{aligned}
& \psi_{3 / 2}^{3 / 2}= \frac{1}{\sqrt{2}}(X+i Y) \alpha, \quad \psi_{1 / 2}^{3 / 2}=\frac{i}{\sqrt{6}}[(X+i Y) \beta-2 Z \alpha], \\
& \psi_{-1 / 2}^{3 / 2}= \frac{1}{\sqrt{6}}[(X-i Y) \alpha+2 Z \beta], \quad \psi_{-3 / 2}^{3 / 2}=\frac{i}{\sqrt{2}}(X-i Y) \beta, \\
& \psi_{1 / 2}^{1 / 2}=\frac{1}{\sqrt{3}}[(X+i Y) \beta+Z \alpha], \\
& \psi_{-1 / 2}^{1 / 2}=\frac{i}{\sqrt{3}}[-(X-i Y) \alpha+Z \beta],
\end{aligned}
$$

where $X, Y$, and $Z$ are the Bloch functions for $\mathbf{k}=0$ that transform under operations of the cubic group as Cartesian coordinates $x, y$, and $z$, respectively. ${ }^{20}$ The $\alpha$ and $\beta$ are the spin functions corresponding to positive and negative spin projections, respectively. The spin-orbit interaction operator is diagonal in this representation, and the coefficients $c_{J, m_{J}}^{\nu}$ are eigenvectors of the Hamiltonian matrix. ${ }^{20}$ The six eigenvalues are degenerate in pairs due to time reversal symmetry. Eigenvalues of the Hamiltonian matrix in representation
(A2) are defined by ${ }^{20}$ three constants of the valence band, $L$, $M$, and $N$, and by the spin-orbit splitting $\Delta$ at $\mathbf{k}=0$. These eigenvalues (for valence electrons since $\alpha<0$ ) are

$$
E_{H}=\frac{F+G}{2}-\frac{\Delta}{3}+\left(\frac{T}{12}-\frac{3 \beta}{T}\right)\left[\alpha+\sqrt{3\left(1-\alpha^{2}\right)}\right],
$$

and

$$
\begin{aligned}
& E_{L}=\frac{F+G}{2}-\frac{\Delta}{3}+\left(\frac{T}{12}-\frac{3 \beta}{T}\right)\left[\alpha-\sqrt{3\left(1-\alpha^{2}\right)}\right], \\
& E_{S}=\frac{F+G}{2}-\frac{\Delta}{3}-\alpha\left(\frac{T}{6}-\frac{6 \beta}{T}\right),
\end{aligned}
$$

where

$$
\begin{aligned}
& F=\frac{L+M}{2}\left(k_{x}^{2}+k_{y}^{2}\right)+M k_{z}^{2}, \\
& G=\frac{F}{3}+\frac{2}{3}\left[M\left(k_{x}^{2}+k_{y}^{2}\right)+L k_{z}^{2}\right],
\end{aligned}
$$

$$
\begin{aligned}
& I=\frac{1}{\sqrt{12}}\left\{(L-M)\left(k_{x}^{2}-k_{y}^{2}\right)-2 i N k_{x} k_{y}\right\} \\
& H=-\frac{N}{\sqrt{3}}\left(k_{y} k_{z}+i k_{x} k_{z}\right) \\
& T=\sqrt[6]{27 \Theta}
\end{aligned}
$$

$$
\begin{aligned}
\Theta= & 36(F-G)^{4}\left[\Delta^{2}+6\left(|I|^{2}+|H|^{2}\right)\right]+432\left(|I|^{2}\right. \\
& \left.+|H|^{2}\right)^{2}\left[3(F-G)^{2}+4\left(|I|^{2}+|H|^{2}\right)\right]+288\left(|I|^{2}\right. \\
& \left.+|H|^{2}\right) \Delta^{2}\left[(F-G)^{2}+2\left(|I|^{2}+|H|^{2}\right)\right]+\frac{1}{9}\left[(F-G)^{2}\right. \\
& \left.+4\left(|I|^{2}+|H|^{2}\right)\right]\left[144 \Delta^{4}+243(F-G)^{4}\right]+\frac{64}{27} \Delta^{6},
\end{aligned}
$$




$$
\alpha=\cos \left(\frac{1}{3} \cos ^{-1}\left\{\frac{\left|-162 \sqrt{3} R-8 \Delta^{3}-27(F-G)\left[(F-G)^{2}+6\left(|H|^{2}-2|I|^{2}\right)\right]\right|}{T^{3}}\right\}\right),
$$

$$
\begin{aligned}
R= & I H^{* 2}+I^{*} H^{2}=\frac{N^{2} k_{z}^{2}}{6 \sqrt{3}}\left[-2(L-M)\left(k_{x}^{2}-k_{y}^{2}\right)^{2}\right. \\
& \left.-8 N k_{x}^{2} k_{y}^{2}\right], \\
\beta= & -\left(\frac{F-G}{2}\right)^{2}-|H|^{2}-|I|^{2}-\frac{\Delta^{2}}{9} .
\end{aligned}
$$

Subscript labels of energies in Eq. (A3) represent heavy band $(H)$, light band $(L)$, and split-off band $(S)$. The positive energies of holes are the negative of Eqs. (A3).

The band constants $L, M$, and $N$ needed to calculate all terms in Eqs. (A3)-(A5) are tabulated for a number of semiconductors in Ref. 31. Light- and heavy-hole constant energy surfaces for silicon have been presented in Refs. 33 and 34 . The energy surfaces of germanium are warped spheres, where the small anisotropy results from weak mixing with the distant $(\Delta=290 \mathrm{meV})$ split-off band. In contrast, the mixing is much stronger for silicon, and a warped spheres description is a poor choice. The strong anisotropy of energy surfaces gives strong direction dependence for the density of hole states, which clearly affects the rates of optical phonon scattering.

Once the hole spectrum $E_{\nu}(\nu=H, L, S)$ has been found, corresponding coefficients $c_{i}^{\nu}$ that determine the hole wave function can be calculated. Since all three distinct eigenvalues [Eq. (A3)] are doubly degenerate, the sets of coefficients $c_{J, m_{J}}^{\nu}$ for each band $\nu$ are determined only to within a unitary transformation of the degenerate functions. The coefficients $c_{J, m_{J}}^{\nu+}$ and $c_{J, m_{J}}^{\nu-}$ of the two degenerate functions $\psi^{\nu+}$ and $\psi^{\nu-}$ for each band $\nu$ may be subjected to the following conditions: $:^{20}$

$$
\begin{aligned}
& c_{3 / 2,3 / 2}^{\nu-}=-c_{3 / 2,-3 / 2}^{\nu+}, \quad c_{3 / 2,-3 / 2}^{\nu-}=c_{3 / 2,3 / 2}^{\nu+}{ }^{*}, \\
& c_{3 / 2,1 / 2}^{\nu-}=c_{3 / 2,-1 / 2}^{\nu+}{ }^{*}, \quad c_{3 / 2,-1 / 2}^{\nu-}=-c_{3 / 2,1 / 2}^{\nu+}{ }^{*}, \\
& c_{1 / 2,1 / 2}^{\nu-}=-c_{1 / 2,-1 / 2}^{\nu+}, \quad c_{1 / 2,-1 / 2}^{\nu-}=c_{1 / 2,1 / 2}^{\nu+}{ }^{*} .
\end{aligned}
$$

This choice of the coefficients [Eq. (A6)] is consistent with the requirement that the eigenvalues [Eq. (A3)] of the Hamiltonian matrix in the basis [Eq. (A2)] are doubly degenerate. The "+" and "-" labels on the band index $\nu$ can be considered as an "effective spin" projection $\sigma$. It is convenient to write the wavefunctions (A1) as a column matrix of expansion coefficients $c_{J, m_{J}}^{\nu^{\sigma}}(\mathbf{k})$ in terms of Luttinger-Kohn functions [Eq. (A2)],

$$
\psi^{\nu^{\sigma}}(\mathbf{k}, \mathbf{r})=\exp (i \mathbf{k} \cdot \mathbf{r})\left\|\begin{array}{c}
c_{3 / 2,3 / 2}^{\nu^{\sigma}} \\
c_{3 / 2,1 / 2}^{\nu^{\sigma}} \\
c_{3 / 2,-1 / 2}^{\nu^{\sigma}} \\
c_{3 / 2,-3 / 2}^{\nu^{\sigma}} \\
c_{1 / 2,1 / 2}^{\nu^{\sigma}} \\
c_{1 / 2,-1 / 2}^{\nu^{\sigma}}
\end{array}\right\|=\exp (i \mathbf{k} \cdot \mathbf{r}) Y^{\nu^{\sigma}}(\mathbf{k})
$$

where the eigenfunctions $\Upsilon^{\nu^{\sigma}}(\mathbf{k})$ that satisfy Eq. (A6) are, for light $(\nu=L)$ and heavy $(\nu=H)$ bands,

$$
\begin{aligned}
& \Upsilon^{H^{+}} \\
& =\|\left(E_{H}-F\right)\left(2 E_{H}+F-3 G\right)-2 \Delta\left(G-E_{H}\right)-3|H|^{2} \\
& 2 H^{*}\left(E_{H}-F+\Delta\right)-2 \sqrt{3} H I^{*} \\
& I^{*}\left(2 E_{H}+F-3 G+2 \Delta\right)-\sqrt{3} H^{* 2} \\
& 0 \\
& i \sqrt{2}\left\{-H^{*}\left(E_{H}-F\right)+\sqrt{3} H I^{*}\right\} \\
& i \sqrt{2}\left\{I^{*}\left(2 E_{H}+F-3 G\right)-\sqrt{3} H^{* 2}\right\}
\end{aligned} \| \text {, }
$$

$$
\Upsilon^{H^{-}} \| \begin{gathered}
0 \\
I\left(2 E_{H}+F-3 G+2 \Delta\right)-\sqrt{3} H^{2} \\
-2 H\left(E_{H}-F+\Delta\right)+2 \sqrt{3} H^{*} I \\
\left(E_{H}-F\right)\left(2 E_{H}+F-3 G\right)-2 \Delta\left(G-E_{H}\right)-3|H|^{2} \\
i \sqrt{2}\left\{I\left(2 E_{H}+F-3 G\right)-\sqrt{3} H^{2}\right\} \\
-i \sqrt{2}\left\{-H\left(E_{H}-F\right)+\sqrt{3} H^{*} I\right\}
\end{gathered}
$$

$$
Y^{L^{+}}=\left\|\begin{array}{c}
-2 H\left(E_{L}-F+\Delta\right)+2 \sqrt{3} H^{*} I \\
\left(E_{L}-F\right)\left(-2 E_{L}+F+G-2 \Delta\right)+4|I|^{2}+|H|^{2} \\
0 \\
-I^{*}\left(2 E_{L}+F-3 G+2 \Delta\right)+\sqrt{3} H^{* 2} \\
i \sqrt{2}\left\{\left(E_{L}-F\right)(G-F)+|H|^{2}-2|I|^{2}\right\} \\
i \sqrt{2}\left\{\sqrt{3} H^{*}\left(E_{L}-F\right)-3 H I^{*}\right\}
\end{array}\right\|,
$$




$$
Y^{L^{-}}=\left\|\begin{array}{c}
I\left(2 E_{L}+F-3 G+2 \Delta\right)-\sqrt{3} H^{2} \\
0 \\
\left(E_{L}-F\right)\left(2 E_{L}-F-G+2 \Delta\right)-4|I|^{2}-|H|^{2} \\
-2 H^{*}\left(E_{L}-F+\Delta\right)+2 \sqrt{3} H I^{*} \\
i \sqrt{2}\left\{\sqrt{3} H\left(E_{L}-F\right)-3 H^{*} I\right\} \\
-i \sqrt{2}\left\{\left(E_{L}-F\right)(G-F)+|H|^{2}-2|I|^{2}\right\}
\end{array}\right\| .
$$

Normalizing these vectors determines the wave functions (A1) for light- and heavy-hole valence bands in the threeband, $6 \times 6$, Luttinger-Kohn representation. Any linear combination of column vectors $Y^{\nu+}$ and $Y^{\nu-}$ for the same band index $\nu$ from Eqs. (A8) and (A9) is also an eigenfunction of the Hamiltonian matrix.

When carrier kinetic energy is small compared to the spin-orbit splitting, the wave function (A1) can be written in a reduced basis consisting of the first four Luttinger-Kohn functions with $J=3 / 2$, namely, $\psi_{3 / 2}^{3 / 2}, \psi_{1 / 2}^{3 / 2}, \psi_{-1 / 2}^{3 / 2}$, and $\psi_{-3 / 2}^{3 / 2}$. In this two-band $4 \times 4$ approximation, the valence band is parabolic and constant energy surfaces in momentum space are warped spheres. The valence electron ( $A$ is negative) energy spectrum in this approximation is ${ }^{20}$

$$
\begin{aligned}
E_{H} & =\frac{F+G}{2}+\sqrt{\left(\frac{F-G}{2}\right)^{2}+|H|^{2}+|I|^{2}} \\
& =A k^{2}+\sqrt{B^{2} k^{4}+C^{2}\left(k_{x}{ }^{2} k_{y}{ }^{2}+k_{x}{ }^{2} k_{z}{ }^{2}+k_{y}{ }^{2} k_{z}{ }^{2}\right)}
\end{aligned}
$$

$$
\begin{aligned}
E_{L} & =\frac{F+G}{2}-\sqrt{\left(\frac{F-G}{2}\right)^{2}+|H|^{2}+|I|^{2}} \\
& =A k^{2}-\sqrt{B^{2} k^{4}+C^{2}\left(k_{x}{ }^{2} k_{y}{ }^{2}+k_{x}{ }^{2} k_{z}{ }^{2}+k_{y}{ }^{2} k_{z}{ }^{2}\right)},
\end{aligned}
$$

$$
E_{S}=\frac{F+G}{2}-\Delta=A k^{2}-\Delta .
$$

Positive hole energies are the negative of Eqs. (A10). The wave functions (A8) and (A9) reduce to

$$
\begin{gathered}
\Upsilon_{3 / 2}^{H+}(\Omega)=\frac{1}{\sqrt{\left(E_{H}-F\right)\left(E_{H}-E_{L}\right)}}\left\|\begin{array}{c}
H \\
E_{H}-F \\
0 \\
I^{*}
\end{array}\right\|, \\
\Upsilon_{3 / 2}^{H-}(\Omega)=\frac{1}{\sqrt{\left(E_{H}-F\right)\left(E_{H}-E_{L}\right)}}\left\|\begin{array}{c}
-I \\
0 \\
-\left(E_{H}-F\right) \\
H^{*}
\end{array}\right\|,
\end{gathered}
$$

$$
\begin{gathered}
\Upsilon_{3 / 2}^{L+}(\Omega)=\frac{1}{\sqrt{\left(E_{L}-F\right)\left(E_{L}-E_{H}\right)}}\left\|\begin{array}{c}
H \\
E_{L}-F \\
0 \\
I^{*}
\end{array}\right\|, \\
\Upsilon_{3 / 2}^{L-}(\Omega)=\frac{1}{\sqrt{\left(E_{L}-F\right)\left(E_{L}-E_{H}\right)}}\left\|\begin{array}{c}
-I \\
0 \\
-\left(E_{L}-F\right) \\
H^{*}
\end{array}\right\|,
\end{gathered}
$$

Numerical values for constants $A, B, C$, and $D$ in Eqs. (A10), (4), and (5) are calculated ${ }^{20}$ from the constants $L, M$, and $N$.

Having presented the wave functions and energy spectrum for both three-band model and two-band approximation, we next consider transitions between states caused by scattering on optical phonons for each case. The optical branch of the phonon spectrum represents the set of harmonic oscillators describing the relative motion of the two individual atoms in a primitive cell. The phonon polarization may be taken as any set of orthogonal unit vectors. ${ }^{13}$ The matrix of the optical deformation operator $H_{\mathrm{opt}}$ in the basis (A2) is ${ }^{20}$

$$
H_{\mathrm{opt}}\left(\mathbf{e}^{s}\right)=\frac{2}{\sqrt{3}} d_{\mathrm{opt}}\left(e_{x}^{s}\left[J_{y} J_{z}\right]+e_{y}^{s}\left[J_{x} J_{z}\right]+e_{z}^{s}\left[J_{x} J_{y}\right]\right),
$$

where $d_{\text {opt }}=d_{0} / a_{0}$, the optical deformation potential $d_{0}$ has the value $40.3(26.6) \mathrm{eV}$ for $\mathrm{Ge}(\mathrm{Si})$, the lattice constant $a_{0}$ is $0.566(0.543) \mathrm{nm}$ for $\mathrm{Ge}(\mathrm{Si})$, and $\mathbf{e}^{s}$ is the phonon $s$-branch polarization vector. The $\left[J_{i} J_{j}\right]$ are the symmetrized products of the matrices of angular momentum components ${ }^{35}$ $J_{i}$ and $J_{j}$ in the basis (A2),

$$
\left[J_{i} J_{j}\right]=\frac{1}{2}\left(J_{i} J_{j}+J_{j} J_{i}\right)
$$

The rate of transitions from state $\left|\mathbf{k}, \nu^{\sigma}\right\rangle$ to state $\left|\mathbf{k}^{\prime}, \nu^{\prime \sigma^{\prime}}\right\rangle$ with absorption (upper part) or emission (lower part) of an optical phonon $\mathbf{q}$ of branch $s$ is

$$
\begin{aligned}
& P\left(\mathbf{k}, \nu^{\sigma} ; \mathbf{k}^{\prime}, \nu^{\prime \sigma^{\prime}}\right)=\frac{2 \pi}{\hbar}\left(\frac{\hbar}{2 \rho \omega_{\mathrm{op}} V_{c}}\right)\left\|\bar{n}_{\hbar \omega_{\mathrm{op}}} \bar{n}_{\hbar \omega_{\mathrm{op}}}+1\right\| \\
& \times\left|\left\langle\mathbf{k}^{\prime}, \nu^{\prime \sigma^{\prime}}\left|H_{\mathrm{opt}}\left(\mathbf{e}^{s}\right)\right| \mathbf{k}, \nu^{\sigma}\right\rangle\right|^{2} \delta\left\{E_{\nu}(\mathbf{k})\right. \\
& \left.-E_{\nu^{\prime}}\left(\mathbf{k}^{\prime}\right) \pm \hbar \omega_{\mathrm{op}}\right\} \text {, }
\end{aligned}
$$

which is subject to the condition

$$
\mathbf{k}^{\prime}=\mathbf{k}^{\prime}\left[E_{\nu}(\mathbf{k}) \pm \hbar \omega_{o p}, \nu^{\prime}, \Omega^{\prime}\right]
$$

In Eq. (A15) $\rho$ is the mass density, $\bar{n}_{\hbar \omega_{\mathrm{op}}}$ is the Bose-Einstein phonon distribution, $H_{\mathrm{opt}}$ is the deformation potential operator, and $V_{c}$ is the volume of the crystal. Integrating over final-state hole wave vector $\mathbf{k}^{\prime}$, summing over three orthogonal phonon polarization vectors, ${ }^{13}$ summing over final effective spin projections, and averaging over initial effective spin projections [see Eq. (2)], we obtain for the total transition rate Eq. (1). 
${ }^{1}$ C. Jacoboni and L. Reggiani, Rev. Mod. Phys. 55, 645 (1983).

${ }^{2}$ T. Brudevoll, T. A. Fjeldly, J. Baek, and M. S. Shur, J. Appl. Phys. 67, 7373 (1990).

${ }^{3}$ B. K. Ridley, Quantum Processes in Semiconductors (Oxford, New York, 1999).

${ }^{4}$ C. Chen, M. Dutta, and M. A. Stroscio, Phys. Rev. B 70, 075316 (2004).

${ }^{5}$ F. M. Bufler, A. Schenk, and W. Fichtner, J. Appl. Phys. 90, 2626 (2001).

${ }^{6}$ D. Chattopadhyay and H. J. Queisser, Rev. Mod. Phys. 53, 745 (1981).

${ }^{7}$ M. V. Dolguikh, A. V. Muravjov, and R. E. Peale, Phys. Rev. B 73, 075327 (2006).

${ }^{8}$ M. V. Dolguikh, A. V. Muravjov, R. E. Peale, M. Klimov, O. A. Kuznetsov, and E. A. Uskova, J. Appl. Phys. 98, 023107 (2005).

${ }^{9}$ M. V. Dolguikh, A. V. Muravjov, and R. E. Peale, J. Appl. Phys. 99, 093106 (2006).

${ }^{10}$ M. V. Dolguikh, A. V. Muravjov, R. E. Peale, R. A. Soref, D. Bliss, C. Lynch, and D. W. Weyburne, Proc. SPIE 5931, 310 (2005).

${ }^{11}$ E. Bründermann, E. E. Haller, and A. V. Muravjov, Appl. Phys. Lett. 73, 723 (1998).

${ }^{12}$ E. V. Starikov and P. N. Shiktorov, Opt. Quantum Electron. 23, S177 (1991).

${ }^{13}$ P. Lawaetz, Phys. Rev. 174, 867 (1968).

${ }^{14}$ E. Bründermann, in Long Wavelength Infrared Semiconductor Lasers, edited by H. K. Choi (Wiley, New York, 2004), pp. 279-343.

${ }^{15}$ V. N. Shastin, Opt. Quantum Electron. 23, S111 (1991).

${ }^{16}$ A. A. Andronov et al., Physica B 134, 210 (1985).

${ }^{17}$ P. Kinsler and W. Th. Wenckebach, J. Appl. Phys. 90, 1692 (2001).

${ }^{18}$ V. I. Gavrilenko and Z. F. Krasil'nik, Opt. Quantum Electron. 23, S323 (1991).

${ }^{19}$ E. V. Starikov and P. N. Shiktorov, Opt. Quantum Electron. 23, S341 (1991).

${ }^{20}$ G. L. Bir and G. E. Pikus, Symmetry and Strain-Induced Effects in Semiconductors (Wiley, New York, 1974).
${ }^{21}$ J. E. Dijkstra and W. Th. Wenckebach, J. Phys.: Condens. Matter 9, 10373 (1997).

${ }^{22}$ F. Szmulowicz, Phys. Rev. B 28, 5943 (1983).

${ }^{23}$ R. Kohler, A. Tredicucci, F. Beltram, H. E. Beere, E. H. Linfield, A. G. Davies, D. A. Ritchie, R. C. Iotti, and F. Rossi, Nature (London) 417, 156 (2002).

${ }^{24}$ Peter Bruesch, Phonons: Theory and Experiments II: Experiments and Interpretation of Experimental Results (Phonons) (Springer, Berlin, 1987), p. 57.

${ }^{25}$ A. A. Andronov, L. S. Masov, and I. M. Nefedov, in Submillimeter Wave Lasers in Semiconductors Using Hot Holes, edited by A. A. Andronov (IAP AS, Gorki, 1986), in Russian.

${ }^{26}$ V. I. Gavrilenko, E. P. Dodin, Z. F. Krasil'nik, and M. D. Chernobrovtseva, Sov. Phys. Semicond. 21, 299 (1987).

${ }^{27}$ L. E. Vorobjev, S. N. Danilov, and V. I. Stafeev, Opt. Quantum Electron. 23, S221 (1991).

${ }^{28}$ A. V. Muravjov, R. C. Strijbos, W. Th. Wenckebach, and V. N. Shastin, Phys. Status Solidi B 205, 575 (1998).

${ }^{29}$ E. Bründermann, E. E. Haller, and A. V. Muravjov, Appl. Phys. Lett. 73, 723 (1998).

${ }^{30}$ L. Reggiani, S. Bosi, C. Canali, and F. Nava, Phys. Rev. B 23, 3050 (1981).

${ }^{31}$ Physics of Group IV Elements and III-V Compounds, edited by O. Madelung, Landolt-Börnstein, New Series, Group III, Pt. A, Vol. 17 (Springer Verlag, Berlin, 1982).

${ }^{32}$ J. M. Luttinger and W. Kohn, Phys. Rev. 97, 869 (1954).

${ }^{33} \mathrm{M}$. Lundstrom, Fundamentals of Carrier Transport, 2nd ed. (Cambridge University Press, Cambridge, U.K., 2000) pp. 16-18.

${ }^{34} \mathrm{~J}$. Singh, Physics of Semiconductors and their Heterostructures (McGrawHill, New York, 1993).

${ }^{35}$ L. D. Landau and E. M. Lifshitz, Quantum Mechanics (Non-relativistic Theory), Course of Theoretical Physics (Pergamon, Oxford, 1977), Vol. 3. 